\title{
The colourful picture of public health
}

\author{
Katrin Olenik • Bosco Lehr
}

Published online: 14 July 2013

(C) Springer-Verlag Berlin Heidelberg 2013

This issue of the Journal of Public Health contains different articles in the area of public health and health sciences. Although each article is, in its difference from the others, very relevant in its very special field, all articles together add to the total picture of public health in the present issue. This is comparable to a colourful picture, which when moving closer, consists of several small photographs, that as a whole make up a mosaic. The content of each single photo can differ highly, as long as in their entirety they make up a nice picture. The same applies to a digital photograph, consisting of many tiny differently coloured pixels, one next to the other, seemingly not having anything in common, but when viewed from the distance, are all equally important to the photograph as a whole.

Some of the articles in this issue are more closely related to each other than others, which is also the case in a photomontage or a pixel collection. In a digital photograph, pixels in orange and yellow are not as different for example as blue and yellow pixels, which analogously applies to the articles in this issue as well. With Thumboo et al. and Tan et al., two research groups have focussed on the impact of smoking on different aspects of life. Working with the same topic their focus on the subject of smoking differs. On the one hand, using interview surveys among multi-ethic participants in Singapore, Thumboo et al. found out that there is an influence of self-reported smoking status on the healthrelated quality of life, while Tan et al. on the other hand, discovered a correlation between smoking cigarettes and the body mass index in Malaysia, analysing data from observations done via the Malaysia non-communicable disease surveillance.

K. Olenik $\cdot$ B. Lehr $(\square)$

Department for eHealth and Health Care Management, Flensburg

University of Applied Sciences, Kanzleistr. 91-93,

24943 Flensburg, Germany

e-mail: lehr@fh-flensburg.de
The similar colours rose and pink could be designated to the articles of Ansari et al. and Diehl et al., each focussing on a narrow group of participants. Ansari et al. analysed the relation between stress and self-reported symptoms and health complaints among 3,706 students. They developed the four subgroups - psychological symptoms, circulatory or breathing symptoms, gastro or intestinal symptoms and pains or aches, by which they found among other findings the highest correlation in stress and a rise in psychological symptoms as well as a correlation between better life quality and less psychological and circulatory/breathing symptoms. The second article concentrating on a special group of population is by Diehl et al. on the usage of sunbeds among minors in Germany in spite of legal bans. This study is based on data from the SUNStudy, focussing on the current research of only the 14- to 17-year-olds, revealing that $8.7 \%$ of the minors in Germany indicated that they had used sunbeds despite the existing legal ban.

Staying with the analogy of a colourful pixel collection, the articles by Ladusingh and Pandey and Rosano et al. could be coloured blue and turquoise, with hospitalization being the linking topic of both. Ladusingh and Pandey explored with the help of data from national representative unit levels in India the cost differences of hospital patients dying in hospitals compared to surviving patients. Using bivariate analysis' and descriptive statistics they discovered that costs for the dying are larger than for surviving patients, especially in rural regions. The other study concerned with hospitalization in this issue is by Rosano et al. comparing hospitalization rates for "ambulatory care sensitive conditions (ACSCs)" in Germany and Italy with a focus on the primary health sectors of both countries. In their analysis, using Poisson regressions, they found out among other findings that the hospitalization rate for chronic ACSCs in southern Italy and eastern Germany is higher than in the respective remaining parts of the two countries.

Rather different colours such as green, purple, black, or red, could be dedicated to the articles of Hussein et al., Leicht et al., Patera and Wild and Lavanya and Ravichandran since, 
although they add to the total picture of public health, they are not related content wise to any of the other articles in this issue.

Hussein et al. focussed on the preventive activities of dentists in Bremen and Lower Saxony (Germany), and found out, analysing 640 postal survey questionnaires, that preventive care is already in use among many dentists, while the provision of counselling on dental risks and behavioural changes could still be enhanced. Another field was investigated by Leicht et al. researching the area of patient satisfaction in the context of quality management with the help of 100 patient questionnaires from before and after the revision of the quality-management tool in specialised medical centers. They came to the conclusion that patient satisfaction did not improve significantly, but that a correlation could be seen in certain quality improvements and a rising patient satisfaction. Patera and Wild used in their research the results from 11 semi-structured telephone interviews with experts from research organisations focussed on public health and health services in the three countries of Norway, the Netherlands and the United Kingdom, to identify the connection between research and national policies and practices. Their study showed that many diverse ways of interaction between research and stakeholders are existent, mainly triggered by research commissioning institutions, with the help of funding or forums to create networks. Another totally different field was dealt with by Lavanya and Ravichandran. They conducted a study of water samples to analyse and compare the microbial contamination status of drinking water from household storages and water pipes in India. Analysing samples from seven sources, they discovered that piped water is less often contaminated than water from household storages.

The wide range of the articles' topics in this issue shows how colourful the field of public health is and how all of these little pieces taken together help to build the whole extendable picture of public health. 